\title{
SULLE FORZE MOLECOLARI INTERNE DEI CORPI SOLIDI E LORO RELAZIONI COLLE PROPRIETÀ ELASTICHE DI ESSI.
}

Nota del.Prof. STEFANO PAGLIANI.

Dal 1895 al 1911 A. Amagat pubblicò una serie di Note sulla pressione nei fluidi '). I risultati principali di tale studio furono i seguenti.

Quando una massa di gas subisce una variazione isotermica di volume $d v$, il lavoro corrispondente è espresso da $\mathrm{T} \frac{d p}{d t} d v$. Se si sottrae il lavoro esterno, resta per il lavoro interno $\left(\mathrm{T} \frac{d p}{d t}-p\right) d v$. Il quoziente del lavoro interno per la variazione di volume sarebbe una pressione interna:

$$
\pi=\mathrm{T} \frac{d p}{d t}-p
$$

Questo ragionamento si può applicare solo alla condizione che il lavoro interno considerato sia esclusivamente quello di forze, all'azione delle quali si può sostituire quella di una pressione esterna, ciò̀ l'attrazione reciproca delle molecole. Ciò porterebbe ad assumere che a temperatura costante il lavoro interno della molecola è nullo, cioè che l'energia molecolare varia solo colla temperatura, ciò che sembra verosimile finchè le distanze delle molecole sono relativamente grandi; per un volume sufficientemente piccolo si comprende che la reazione delle molecole compresse le une contro le altre, quando si fa

1) Notes de Physique et de thermodynamique. - Sur la pression interieure dans les fuides. 1911. 
variare questo volume, possa dar luogo, anche a temperatura costante, ad un lavoro intermolecolare.

Secondo Amagat si può domandare se una parte di questo lavoro non sia dovuto ad uno dei fattori della pressione interna effettiva, e rientra quindi nella somma, il cui quoziente per $d v$ da il valore effettivo di questa pressione.

Amagat introduce poi un'altra pressione interna $\pi^{\prime}$, il cui valore si deduce ponendo il viriel delle forze interiori di Clansius, da cui deve dipendere evidentemente il valore della pressione interna, $W={ }_{2}^{3} \pi^{\prime} v$.

Sarrau ha dimostrato che nel caso, in cui il volume delle molecole e l'ampiezza dei movimenti stazionari siano piccolissimi rispetto alle distanze intramolecolari, il prodotto $\pi^{\prime} d v$ rappresenterebbe il lavoro intermolecolare relativo alla variazione di volume $d v$. Egli mette l'equazione del calore elementare $d q$ sotto la forma

$$
d q=\mathrm{M} d t+\mathrm{A}\left(p+\pi^{\prime}\right) d v
$$

ma da essa si deduce per $\pi$ ' la stessa espressione (1) trovata per $\pi$ e ciò̀ $\pi=\pi$ '. Invece $\mathrm{i}$ calcoli di Amagat hanno dimostrato che i valori di $\pi$ e di $\pi$ ' sono estremamente differenti per uno stesso gas, a tutte le pressioni. Quindi Amagat conchiude che o la costituzione dei fluidi non risponde in alcun modo all'ipotesi fatta, oppure le basi stesse della teoria sono inapplicabili a questa classe di corpi, e ciò per tutte le pressioni.

Sarrau aveva, invero, già fatto notare che si è ai solidi che i risultati, ai quali potrebbe condurre l'ipotesi in questione, sembrerebbero avere la maggior probabilita di essere applicabili, poichè in questi corpi le molecole oscillano probabilmente assai poco attorno a certe posizioni di equilibrio.

Amagat in seguito dimostrò che $\pi$ non corrisponde affatto alla nozione di pressione interna definita più sopra, poichè una parte del lavoro $\pi d v$ non dipende punto dalle azioni, che possono avere per effetto una variazione di volume; $\pi$ ' al contrario non dipende che da queste azioni; essa è per la sua definizione 
stessa del tutto indicata come pressione interiore, da introdursi nell'equazione di stato dei gas.

Infine per eliminare la contraddizione derivante dalle differenze fra $i$ valori numerici delle funzioni $\pi$ e $\pi^{\prime}$ Amagat introduce per i gas un'altra ipotesi, quella di forze interne speciali, che producano la imperfezione del fluido, e di una pressione interiore totale

$$
\Pi^{\prime}=\pi^{\prime}+\pi^{\prime \prime}=\frac{p_{0} v_{0}}{v}-p
$$

I valori di $\pi^{\prime \prime}$ risultano tutti negativi e sotto volume costante $\dot{\pi}$ " è proporzionale alla temperatura assoluta; essa agisce contro l'attrazione molecolare e la pressione esterna e tende quindi ad aumentare il volume. Amagat suppone che sia dovuta alla esistenza di un fluido perfetto, che riempie il volume intramolecolare. Esso deve formare delle piccole atmosfere di densità decrescente a partire dalla regione centrale attorno a ciascun aggruppamento molecolare.

Amagat infine conchiude che per distanze intramolecolari piccolissime, sembra naturale che si debba tener conto delle variazioni della energia intermolecolare col volume; ma si può concepire un' ipotesi mista nella quale interverebbero simultaneamente l'influenza delle atmosfere e quella dell'energia intermolecolare.

Quanto ai corpi solidi l'unico tentativo di calcolare una pressione interna in essi fu fatto da Th. W. Richards ${ }^{1}$ ), volendosene servire in appoggio della sua ipotesi sulla comprimibilita degli atomi. Egli la calcolò mediante l'espressione $\mathrm{P}=\frac{\mathrm{C} d t}{\mathrm{~V}} \frac{d}{d v}$ in cui $\mathrm{C}$ è il calore atomico espresso in Joule, $\mathrm{V}$ il volume atomico. La pressione risulta quindi riferita all'unità di volume. Però il calcolo non è esatto perchè fondato sul lavoro della dilatazione a pressione costante, il quale dipende

') Proc. Amer. Acad., 1903, XXXVII. 
sia dalla prossione interna, a calcolarsi, sia dalla esterna, che il Richards ritiene trascurabile in confronto della prima.

Possiamo invece considerare tre specie di forze molecolari interne nei solidi. Anche per essi possiamo supporre una pressione interna, quella che, secondo Amagat e Sarrau, entrerebbe nella espressione del lavoro intermolecolare relativo alla variazione di volume. Nella espressione del calore elementare possiamo introdurre una pressione $\pi$ oltre alla pressione esterna, o scriverla sotto la forma $c_{p} d t=c_{v} d t+\mathrm{A}(p+\pi) d v$.

Applicando questa espressione ad una trasformazione finita

$$
c_{p}=c_{v}+\operatorname{A} \gamma v(p+\pi) \quad \text { da cui } \frac{c_{p}}{\mathrm{~A} \gamma v}=\frac{c_{v}}{\mathrm{~A} \gamma v}+(p+\pi)
$$

possiamo considerare due forze molecolari interne, oltre la pressione esterna.

1. - Una forza molecolare data dall'espressione

$$
\mathrm{F}=\frac{\boldsymbol{c}_{v}}{\mathrm{~A} \boldsymbol{\gamma}^{v}}
$$

essendo A l'equivalente termico dell' unità di lavoro e $\gamma$ il coefflciente di dilatazione eubica per 1. Essa si può considerare come uno dei fattori del lavoro intermolecolare dovuto alle azioni fra le molecole compresse le une rispetto alle altre. Si potrebbe a questa forza dare il nome di forza molecolare di coerenza (Coulomb), e sarebbe una forza di attrazione, che si esercita fra le ultime particelle di un corpo, e a distanze infinitamente piccole. Sarebbe la forza da vincere nell'aumentare la energia cinetica di dette particelle, la loro velocità, l'ampiezza delle loro vibrazioni, rimanendo costante il volume del corpo.

2. - Una forza, analoga per gli effetti alla pressione esterna, e che si può considerare come una pressione interna, - di cui abbiamo gia sopra dato il significato. Essa verrebbe data dall'espressione

$$
\pi=\frac{e_{p}-c_{v}}{A \gamma v}-p
$$


Combinandola coll'espressione di W. Thomson:

$$
c_{p}-c_{v}^{*}=\mathrm{AT} \frac{\gamma^{\mathbf{2}} v_{0}^{2}}{\mu v}
$$

in cui $\mu$ è il coefficiente di compressibilità cubica, si arriva alla espressione:

$$
\pi=\mathbf{T} \frac{\gamma}{\mu\left(1+\gamma_{m} t\right)^{2}}-p
$$

in cui $t$ è la temperatura centigrada corrispondente alla assoluta $\mathbf{T}, \Theta \gamma_{m}$ il coefficiente di dilatazione cubica medio fra $0^{0}$ e $t$.

Per le temperature di $0^{\circ}$ e prossime a questa si può scrivere semplicemente

$$
\pi_{0}=\mathbf{T} \frac{\gamma}{\mu}-p .
$$

Siccome d'altra parte $\mathbf{F}=\frac{c_{p}}{\mathrm{~A} \gamma v}-(\pi+p)$ così risulta:

$$
\mathbf{F}=\frac{c_{p}}{\mathbf{A} \gamma v}-\mathbf{T} \frac{\gamma}{\mu\left(1+\gamma_{m} t\right)^{2}}
$$

e per temperature prossime a $0^{\circ}$ :

$$
\mathrm{F}=\frac{c_{p}}{\mathrm{~A} \boldsymbol{\gamma} v}-\mathrm{T} \frac{\gamma}{\mu}
$$

La espressione (5) corrisponde alla (1) e questa si può per i corpi solidi applicare anche nel caso di trasformazione isotermica con cambiamento di stato di aggregazione, nel passaggio dalla fase solida alla liquida; quindi detta forza molecolare interna, oltre che dall'espressione $\mathrm{T} \frac{d p}{d t}-p$ viene data anche dal quoziente di detto lavoro interno, equivalente al calore interno di fusione, per la variazione di volume, e ciò̀ da

$$
f=\frac{r}{\mathrm{~A} \Delta} \boldsymbol{v}-\boldsymbol{p}
$$

essendo $r$ il calore di fusione totale, $\Delta v$ la variazione di volume della unità di peso, e $p$ la pressione esterna. 
Wsporro ora i risultati del calcolo di queste diverse espressione delle tre forze molecolari interne considerate.

\section{Valori di $F$ e di $\pi$.}

Furono calcolati colle espressioni (6) e (4) per la temperatura di $20^{\circ}$. I dati per i calori specifici a pressione costante sono stati tolti dalle stesse fonti, che mi hanno servito per lo studio sull'entropia '). I valori dei coefficienti di dilatazione cubica ho dedotto dai coefficienti di dilatazione lineare, dovuti per la maggior parte alle misure del Fizeau. I valori dei coefficienti di compressibilità cubica sono quelli determinati da W. Richards e suoi allievi '). Sopra questi dati ho pure calcolato i valori del rapporto $\frac{c_{p}}{c_{v}}$ che qui sotto riporto con gli altri nella seguente tabella:

1) Nuovo Cimento (6) $X, 1915$.

2) Amer. Chem. Soc., 1904, XXVI, 1909, XXXI. - Pub. Carnegie Inst. Wask., 1907, LXI, LXXVI. 
SULLA FORZE MOLECOLARI INIERNE DEI CORPI ECO. 109

\begin{tabular}{|c|c|c|c|c|c|c|c|}
\hline METALL & & & $\frac{c_{p}}{c_{v}}$ & $\gamma \times 10^{6}$ & $\begin{array}{l}\mu \times 10^{\circ} \\
\text { p. megb }\end{array}$ & $\begin{array}{c}\mathbf{F} \\
m e g / \mathrm{em}^{2}\end{array}$ & $\begin{array}{c}\pi \\
m e g / \mathrm{em}^{2}\end{array}$ \\
\hline Potassio & - & & 1,072 & 211,2 & 31,5 & 26720 & 1960 \\
\hline Sodio . & - & & 1,056 & 192,8 & 15,4 & 56470 & 3670 \\
\hline Litio. . & - & & 1,053 & 157,2 & 8,8 & 93665 & 5235 \\
\hline Piombo. & . & & 1,071 & 86,3 & 2,2 & 156580 & 11490 \\
\hline Tallio . & . & & 1,062 & 83,8 & 2,6 & 169170 & 9445 \\
\hline Cadmio . & • & - & 1,070 & 90,1 & 1,9 & 206950 & 13900 \\
\hline Magnesio & - & - & 1,043 & 82,9 & 2,7 & 217430 & 8990 \\
\hline Stagno . & - & - & 1,049 & 64,9 & 1,7 & 240850 & 11190 \\
\hline Zinco - & . & • & 1,056 & 85,0 & 1,5 & 308060 & 16610 \\
\hline Alluminio & - & $\cdot$ & 1,106 & 63,9 & 1,3 & 338900 & 14420 \\
\hline Argento & - & • & 1,040 & 57,9 & 0,84 & 406300 & 20200 \\
\hline Oro . & - & • & 1,045 & 43,3 & 0,47 & 578110 & 26985 \\
\hline Rame . & . & - & 1,041 & 49,1 & 0,54 & 669060 & 26615 \\
\hline Palladio & - & - & 1,032 & 33,1 & 0,38 & 812820 & 25540 \\
\hline Platino . & - & • & 1,032 & 26,8 & 0,21 & 1018860 & 37340 \\
\hline Ferro . & - & - & 1,020 & 34,9 & 0,40 & 1026584 & 25555 \\
\hline $\begin{array}{l}\text { Nichelio } \\
\text { NON META }\end{array}$ & LLI & & 1,041 & 37,5 & 0,27 & 1030395 & 40695 \\
\hline Fosforo & . & - & 1,184 & 375,0 & 20,3 & 29325 & 5415 \\
\hline Iodio. . & . & ${ }^{\circ}$ & 1,145 & 251,1 & 13,0 & 39005 & 5655 \\
\hline Solfo. . & $\bullet$ & $\theta^{\circ}$ & 1,079 & 162,3 & 12,5 & 62860 & 3804 \\
\hline Antimonio & & - & 1,025 & 50,7 & 2,2 & 259200 & 6750 \\
\hline Bismuto & - & $e^{\circ}$ & 1,152 & 39,6 & 2,8 & 308005 & 4144 \\
\hline $\begin{array}{r}\text { Carbonio } \\
\text { mante) }\end{array}$ & & & 1,002 & 2,66 & 0,5 & 6260340 & 1560 \\
\hline
\end{tabular}


Dai valori del rapporto $\frac{c_{p}}{c}$ risulta il valore medio $\mathbf{1 , 0 6 4}$ per 23 elementi solidi (variando fra 1,002 e 1,184), molto inferiore a quello che si calcola teoricamente, $\%$, ammettendo che si verifichi per tutti gli elementi solidi alla temperatura ordinaria la legge di Dulong e Petit, che il calore atomico sia nguale a 6 , e che $i$ metalli solidi siano monoatomici. Invece ho dimostrato in altra nota che quella legge si verifica a differenti temperature per $i$ diversi corpi solidi ').

Il minimo valore di quel rapporto spetta all'elemento, che maggiormente si scosta da detta legge a temperatura ordinaria, e pure è concordante col valore 1 , che si calcola colla formola Nernst-Lindemann, in base alla frequenza del moto vibratorio atomico.

Come si vede, in generale $i$ valori di $F$ risultano in ordine di grandezza inverso di quelli di $\gamma$ e di $\mu$; il che sta a conferma del significato fisico sopra assegnato alla detta forza molecolare. Difatti per la compressione quanto maggiore è lá coerenza, colla quale stanno compresse fra loro le molecole di un corpo, minore sarà l'effetto prodotto da una pressione esterna, che a quella forza si aggiunga. Non si può parlare di proporzionalità numerica, poichè sul valore di $F$ deve influire anche la natura chimica del corpo. Noi vediamo infatti nella serie dei corpi raggruppati fra loro vicini corpi, che presentano delle analogie chimiche, così $\mathrm{K}, \mathrm{N} a, \mathrm{~L} i-\mathrm{Pb}$ e $\mathrm{T} l-\mathrm{C} d$ e $\mathrm{Mg}-$ $\mathbf{A g}, \mathbf{A} u, \mathbf{C} u, \mathbf{P} d, \mathrm{P} t-\mathrm{F} e$ e $\mathrm{N} i-\mathrm{S} b$ e $\mathrm{B} i$.

Quanto alla pressione interna vedremo in seguito, considerando la influenza della temperatura sul suo valore, che per uno stesso corpo essa va diminuendo col diminuire della temperatura, come i dù coefficienti $\gamma$ e $\mu$.

Ciò confermerebbe anche il significato dato sopra a detta forza; poichè tendendo ad annullarsi ogni energia nel corpo deve an-

1) Gazz. Chim. Ital., XLV, 1915. 
che tendere a zero la pressione interna, mentre può sussistere la forza di coerenza.

\section{Relazioni colle proprietà elastiche dei solidi.}

Considerando la forza di coerenza come agente fra 10 ultime molecole di un solido a distanze infinitamente piccole, essa deve avere una funzione importante nei fenomeni di deformazione elastica, dovuta ad azioni meccaniche, ed in cui avviene uno spostamento relativo delle molecole stesse. Essa rappresenterebbe come una resistenza, che si oppone ai cambiamenti di forma, anche non accompagnati da cambiamenti di volume, del solido. Si devono quindi trovare delle relazioni fra $\mathrm{i}$ valori di F e quelli delle grandezze, che si considerano nello studio e nelle applicazioni delle proprietà elastiche dei solidi, come vado a dimostrare per i metalli, per i quali si hanno i dati sperimentali necessari, e che sono anche quelli, che più interessano la tecnica.

Nelle successive colonne della seguente tabella sono indicati i valori di: $\mathrm{F}$, forza molecolare di coerenza, come sopra calcolata;

E, modulo di elasticità per trazione o di Young. Valori dedotti specialmente dalle misure del Wertheim, o riportati nei trattati di fisica e di meccanica;

$n$, modulo di elasticità per torsione o di rigidità. Valori determinati dal Pisati ');

$\mathrm{N}$, modulo di scorrimento o di elasticità tangenziale. Valori determinati da diversi autori ${ }^{2}$ ).

Le quattro grandezze sono espresse in megadine per $\mathrm{mm}^{2}$, per averle dello stesso ordine di grandezza dei valori più comunemente dati nella tecnica in $\mathrm{Kg} / \mathrm{mm}$.

1) Nuovo Cimento (3) I, 1877, IV, 1878, V, 1879.

2) Chwolson. Traitè de Physique, vol. I. 


\begin{tabular}{|c|c|c|c|c|c|c|}
\hline \multirow[t]{2}{*}{ METALLI } & \multirow[t]{2}{*}{$\underset{\mathrm{meg} / \mathrm{mm}^{2}}{\mathbf{F}}$} & \multicolumn{2}{|c|}{$\underset{m e g / m e g / m^{2}}{\mathbf{E}} \underset{\mathrm{E}}{\mathbf{m}}$} & \multicolumn{2}{|c|}{$\begin{array}{c}n \\
m e g / m m^{\circ}\end{array}$} & \multirow[t]{2}{*}{$\begin{array}{c}\mathrm{N} \\
m e g / m m^{2}\end{array}$} \\
\hline & & ricotto & crudo & ricotto & orudo & \\
\hline Piombo . & 1566 & 1692 & 1764 & - & - & - \\
\hline Stagno . & 2408 & - & 4514 & - & - & 1512 \\
\hline Alluminio & 3389 & - & 6970 & - & 1130 & 2283 \\
\hline Argento . & 4063 & 6998 & 7200 & 1247 & 1554 & 2597 \\
\hline Rame. & 6690 & 10308 & 12200 & 1936 & 2125 & 4129 \\
\hline Platino & 10188 & 15208 & 16703 & 3068 & 3240 & - \\
\hline Ferro . & 10266 & 16778 & 20453 & 3973 & - & 6572 \\
\hline Nichel & 10304 & - & 22030 & - & - & - \\
\hline
\end{tabular}

È noto come il paragone fra le proprietà fisiche e meccaniche, non solo di corpi diversi, ma anche di uno stesso corpo mediante dati sperimentali, ottenuti con campioni diversi, offre dell' incertezza.

Due campioni di uno stesso corpo difficilmente sono di composizione perfettamente identiča, ma per di più ciascun campione, non solo difficilmente è perfettamente isotropo, come esigerebbe il detto confronto, ma è stato precedentemente sottoposto ad azioni, che ne hanno modificato in modo speciale la struttura, e particolarmente le proprietà elastiche; ogni campione ha cioè una propria storia, che si dovrebbe conoscere; $\theta$ cid vale specialmente per $i$ corpi, che presentano fenomeni di isteresi nelle loro deformazioni elastiche. Si sa per esempio che un metallo quanto è meglio ricotto, tanto più si presenta omogeneo nella sua struttura; quindi il grado di omogeneità, di isotropia, dipende specialmente dalla sua condizione attuale di tempra o di ricottura, e le sue proprietà elastiche dipendono dal grado di omogeneità; il valore delle costanti di elasticita dipende dal momento della storia del metallo. Basta 
ricordare a questo riguardo i risultati, per non accennare ad altri, delle ricerche del Pisati, che hanno completato e corretto quelle del Wertheim, di quelle del Cantone, cosi estese sopra la isteresi elastica, di quelle del Guidi sull' influenza di sforzi moderati sulle proprietà elastiche dei metalli.

Come si rileva dalla precedente tabella, i valori dei diversi moduli di elasticita risultano disposti nella stessa successione di ordine di grandezza che i valori di F. Ed alla stessa conclusione generale si arriva se si confrontano detti valori con quelli delle altre grandezze, caratterizzanti le proprieta elastiche dei diversi metalli, cioè limiti di elasticità, moduli di solidità o tenacità, per rottura lenta o repentina, che per brevità non ho riportato.

A relazioni numeriche semplici però si arriva soltanto fra i valori della forza molecolare di coerenza e le grandezze che si riferiscono a deformazioni elastiche, nelle quali si può ammettere che si abbiano soltanto degli spostamenti delle molecole fra loro, ma non variazioni nelle distanze relative. Tali sono il modulo di scorrimento e quello di rigidita.

Per definire il modulo di scorrimento consideriamo una. coppia di piani paralleli in un solido, ed una retta flsica normale ai detti piani.

Se applichiamo una forza tangenziale $\mathbf{P}$ sopra ano di essi, la retta fisica gira di un certo angolo $\omega$ per lo scorrimento di un piano sull'altro. Indicando con $\mathrm{N}$ il modulo di scorrimento, esso vien dato da $\mathrm{N}=\frac{\mathrm{P}}{\omega}$. Supponiamo ora di applicare una forza tangenziale di grandezza uguale alla forza intermolecolare di coerenza propria del solido, e calcoliamo l'angolo di rotazione di quella retta fisica mediante $\mathrm{i}$ valori di $\mathrm{N}$, otteniamo i seguenti risultati :

\begin{tabular}{|c|c|c|c|c|c|c|c|}
\hline Stagno . & .. & · & 1,59 & Rame & & & \\
\hline Alluminio & . & & 1,48 & Ferro & - & . & \\
\hline Argento & 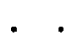 & & 1,56 & & & & \\
\hline
\end{tabular}


Vediamo che $i$ valori di detto rapporto poco differiscono fra loro e tendono verso il valore 1,57 , corrispondente ad una variazione angolare di $90^{\circ}$, essendo il loro valore medio $\mathbf{1 , 5 4}$.

Per definire il modulo di rigidità consideriamo la deformazione, prodotta in un cubo di lato uno, da forze uguali tangenziali, applicate a due coppie di faccie, in direzione parallela alla terza coppia. Se noi immaginiamo condotti i due piani diagonali, le risultanti di quei sistemi di forze saranno una pressione perpendicolare ad uno dei piani diagonali, ed una tensione perpendicolare all'altro. L'effetto sul cubo sarà che esso verrà deformato in un romboedro, cioè una coppia di faccie subira un certo allungamento, l'altra coppia un uguale accorciamento $\theta$ la terza rimarrà inalterata, come pure il volume del solido. Considerando una sezione quadrata, questa si trasforma in un rombo, e la rigidità del solido isotropo, di forma cubica, ossia la resistenza a cambiare di forma sotto un dato sforzo, è direttamente proporzionale alla forza tangenziale unituria, ed inversamente alla variazione di uno degli angoli del cubo. Quindi indicando con $n$ il modulo di rigidità, con $\mathbf{P}$ la forza tangenziale, e con $\theta$ la variazione angolare, si avrd

$$
n=\frac{\mathbf{P}}{\bar{\theta}} \text {. }
$$

Se ora supponiamo di applicare una forza tangenziale di grandezza uguale alla forza intermolecolare di coerenza, e calcoliamo la variazione prodotta mediante $i$ valori di $n$ otteniamo i seguenti risaltati:

$$
\begin{aligned}
& \text { Alluminio • • } \quad 3,00 \\
& \text { Argento . . . } 2,61 \text { a } 3,26 \\
& \text { Rame . . . . } 3,15 \text { a } 3,45 \\
& \text { Ferro . . . . 2,58 } \\
& \text { Platino . . . } 3,14 \text { a } 3,32 \text {. }
\end{aligned}
$$

Questi valori, se si tiene anche qui conto delle condizioni meccaniche eventualmente diverse dei metalli, su cui sono stati 
determinati i valori di $n$, poco differiscono fra loro e tendono verso il valore 3,15 , (essendo il loro medio 3,06 ) corrispondente ad una variazione angolare di $180^{\circ}$.

Sembra quindi possano scriversi con molta approssimazione le due relazioni : $\mathrm{F}=\pi n, \quad \mathrm{~F}=\frac{\pi}{2} \mathrm{~N}$,

(9) che confermano quella già nota

$$
\mathrm{N}=2 n \text {. }
$$

Applicando dette relazioni alla espressione che ci dà il modulo di scorrimento $\mathrm{N}$ mediante il modulo $\mathrm{E}$ ed il coefficiente di Poisson $\sigma$.

(10) $\quad \mathrm{N}=\frac{\mathrm{E}}{2(1+\sigma)} \quad$ si arriva al rapporto $\mathrm{E}=\frac{4(1+\sigma)}{\pi}$.

Calcolando $i$ valori dell' ultimo rapporto, e mediante i valori di $\sigma$ dovuti a Cardani ed a Bock, e paragonandoli con quelli ottenuti direttamente dai valori di $\mathrm{E}$ e di $\mathrm{F}$ si ottiene

\begin{tabular}{|c|c|c|c|c|c|c|}
\hline & & & & $\sigma$ & $\frac{\mathbf{E}}{\overline{\mathbf{F}}}$ & $m$ \\
\hline Piombo. & . & . . & . & 0,375 & 1,08 a 1,12 & 1,75 \\
\hline Alluminio & . & - . & • & 0,373 & 2,06 & 1,74 \\
\hline Argento & - & . . & • & 0,346 & 1,72 a 1,77 & 1,71 \\
\hline Rame. & . & . . & • & 0,348 & 1,54 a 1,82 & 1,71 \\
\hline Ferro . & - & . . & . & 0,321 & 1,63 a 1,99 & 1,68 \\
\hline Nichel . & . & . . & . & 0,329 & 2,13 & 1,69 \\
\hline
\end{tabular}

Come si vede, i valori di $m$ differiscono poco fra loro, il Ioro medio è 1,71. Quelli di ${ }_{\mathrm{F}}^{\mathrm{E}}$ differiscono maggiormente, ma gi deve notare che il valore di $F$ dipende grandemente dalle condizioni, da cui dipendono in generale le proprietà elastiche 
dei solidi, però come medio risulta pure 1,70. Si può quindi ritenere verificata la (10).

$\mathrm{E}$ scrivere $\mathrm{E}=1,70 \mathrm{~F}$ ed approssimativamente

$$
\mathbf{E}={ }_{3}^{5} \mathrm{~F} \text {. }
$$

Hans Lorenz ') giunse per un corpo isotropo, nel quale si ammette che le costanti di elasticità abbiano lo stesso valore in tutte le direzioni, alla seguente relazione fra il modulo $\mathbf{N}$, il modulo $\mathbf{E}$ ed il fattore di contrazione trasversale $\varphi$, reciproco del coefficiente di Poisson :

$$
\varphi=\frac{2 \mathrm{~N}}{\mathrm{E}-2 \mathrm{~N}}
$$

Essendo $\varphi$ sempre positivo ne dedusse che $\mathrm{E}>2 \mathrm{~N}$. Possiamo infatti dalle nostre relazioni $(9$, e $(11$ ricavare $\mathrm{E}=1,33 \times 2 \mathrm{~N}$, inferiore a $3 \mathrm{~N}$, valore cui si giunge nel caso di liquidi, perfettamente incompressibili, ma non elastici, per i quali $\varphi=2$. Per i metalli e leghe $i$ valori di $p$ stanno fra 3 e 4 . Se noi sostituiamo ad E nella (12) l'espressione $2,66 \mathrm{~N}$ otteniamo $\varphi=3$.

Potendosi considerare la (12) come un criterio per l'isotropia di un solido possiamo ritenere $\varphi=\frac{1}{\sigma}=3$, e quindi $\sigma=\frac{1}{3}$, come costanti caratteristiche di un solide isotropo. Ne deriva che essendo il rapporto fra la velocità di propagazione delle onde longitudinali in un corpo elastico e quella delle onde trasversali nella stessa direzione data da $\sqrt{\frac{2(f-1)}{\varphi-\overline{2}}}$, esso risulta uguale a 2 , invece che a $\sqrt{3}$, come risulta adottando $\varphi=4$, secondo Poisson, valore, che non è confermato dai risultati sperimentali.

Applichiamo ora l'espressione $\mathrm{E}=1,70 \mathrm{~F}$ a quella del modulo di compressione uniforme o modulo di elasticita cubica

$$
K=\frac{E}{3(1-2 \sigma)}
$$

4) Lehrbuch dev Techn. Physik, IV, p. 24, 652, 1913. 
per calcolare il coefficiente di compressibilità cubica colla formola

$$
\mu=\frac{3(1-2 \sigma)}{1,70 \mathrm{~F}}
$$

Ho ottenuto i seguenti risultati, che confronto con quelli sperimentali di Grineisen '), di Amagat, e di Richards.

\begin{tabular}{|c|c|c|c|c|c|c|}
\hline & & \multicolumn{4}{|c|}{$\mu \times 10^{\prime}$ per megabar } & \\
\hline & & calcolato & & trova & i sperimenta & meute \\
\hline Piombo . & . & 2,80 & 2,76 & (R.) & 3,2 (G.) & 2,2 (R.) \\
\hline Alluminio & . . & 1,43 & 1,50 & (G.) & $\mathbf{1 , 3 0}$ (R.) & \\
\hline Argento & .. & 1,33 & 0,84 & (R.) & $0,78 \quad(G)$. & \\
\hline Rame & . : & 0,80 & 0,79 & $\left(G_{.}\right)$ & 0,86 (A.) & 0,54 (R.) \\
\hline Ferro & . & 0,62 & 0,64 & (G.) & $0,40 \quad$ (R.) & \\
\hline Nichel & .. & 0,58 & 0,27 & $(\mathbf{R} \cdot)$ & & \\
\hline
\end{tabular}

Tenuto conto del particolar genere di misura, per cui per taluni metalli abbiamo maggiori differenze fra $i$ valori trovati da due sperimentatori, che non fra questi ed i calcolati, possiamo dire che si ha in generale una buona concordanza fra i valori trovati e quelli calcolati con la (2). Per l'argento si può spiegare la notevole differenza con una maggior quantità di rame esistente nelle leghe sperimentate per la compressi. bilità, tendendo quella ad abbassare questa. Quindi si può considerare come sufficientemente approssimata la seguente espressione del modulo di elasticità cubica:

$$
K=\frac{5 F}{9(1-2 \sigma)}
$$

1) Ann. Physik, 33, 1910.

Scrie VI, Vol. XV 


\section{Infiuenza della temperatura.}

La espressione (6) indica come debba variare il valore di F al variare della temperatura. Questa variazione dipende principalmente da quella del rapporto fra il calore specifico a pressione costante ed il coefficiente di dilatazione cubica; coll'aumentare di detto rapporto aumenta il valore di $\mathbf{F}$ e reciprocamente. Dobbiamo inoltre osservare che nel primo termine della (6) entra il calore specifico, che, come si sa, va decrescendo rapidamente a hasse temperature. ì vero che va de. crescendo con queste anche $\gamma$ ed il volume specifico, come pure il secondo termine dell'espressione, sebbene $\mu$ vada decrescendo pur esso. E quindi da attendersi che il valore di $F$ debba presentare dei massimi e dei minimi, come dimostrano infatti i risultati, che si espongono in seguito.

Solo per sei metalli, i principali però, abbiamo i dati sperimentali necessari per calcolare $F$ a diverse temperature. Per alluminio e argento abbiamo le determinazioni del coefficiente di dilatazione lineare a basse temperature di Ayres o Simpson '); per il platino, delle formole sperimentali, fra le quali quella di Kammerlingh Onnes e Clay (1907) per 1'intervallo fra $-183^{\circ}$ e $16^{\circ}$. Per piombo, rame e ferro le determinazioni di Dorsey $\left.{ }^{2}\right)$, Per alluminio, argento, piombo e rame, per la temperatura di $-190^{\circ}$, i valori di $\gamma$ ottenuti da C. L. Lindemann ${ }^{3}$ ). Per temperature superiori all'ordinaria specialmente i valori dovuti al Fizeau.

Per i coefficienti di compressibilità abbiamo le misure fatte alle tre temperature, $-190^{\circ}, 17^{\circ}, 100^{\circ}$, per alcuni metalli più importanti, da E. Grüneisen ${ }^{4}$ ). I suoi valori sono per lo più maggiori di quelli di Richards e suoi allievi. Trattandosi di un confronto generale fra $i$ valori di $F$ e $\pi$ per i corpi sem-

1) Phys Rew., 20, 1905.

) Phys. Rew., 30, 1907, 1908

') Plyys. Zeits. XII, 1911.

4) Loc. cit. 
plici, li ho calcolati mediante i secondi valori, tanto più che la influenza di piccole differenze nei valori di $\mu$ è appena sensibile nella quarta e quinta cifra dei valori di F. Trattandosi invece di studiare la variazione di questi colla temperatura ho adottato i valori di Grüneisen e da essi calcolati per interpolazione $\mathrm{i}$ valori di $\mu$ alle singole temperature, per le quali disponevo pure dei valori di $c$ e di $\gamma$.

Per i calori specifici mi servii delle fonti già citate.

Riporto $\mathrm{i}$ risultati nella tabella seguente:

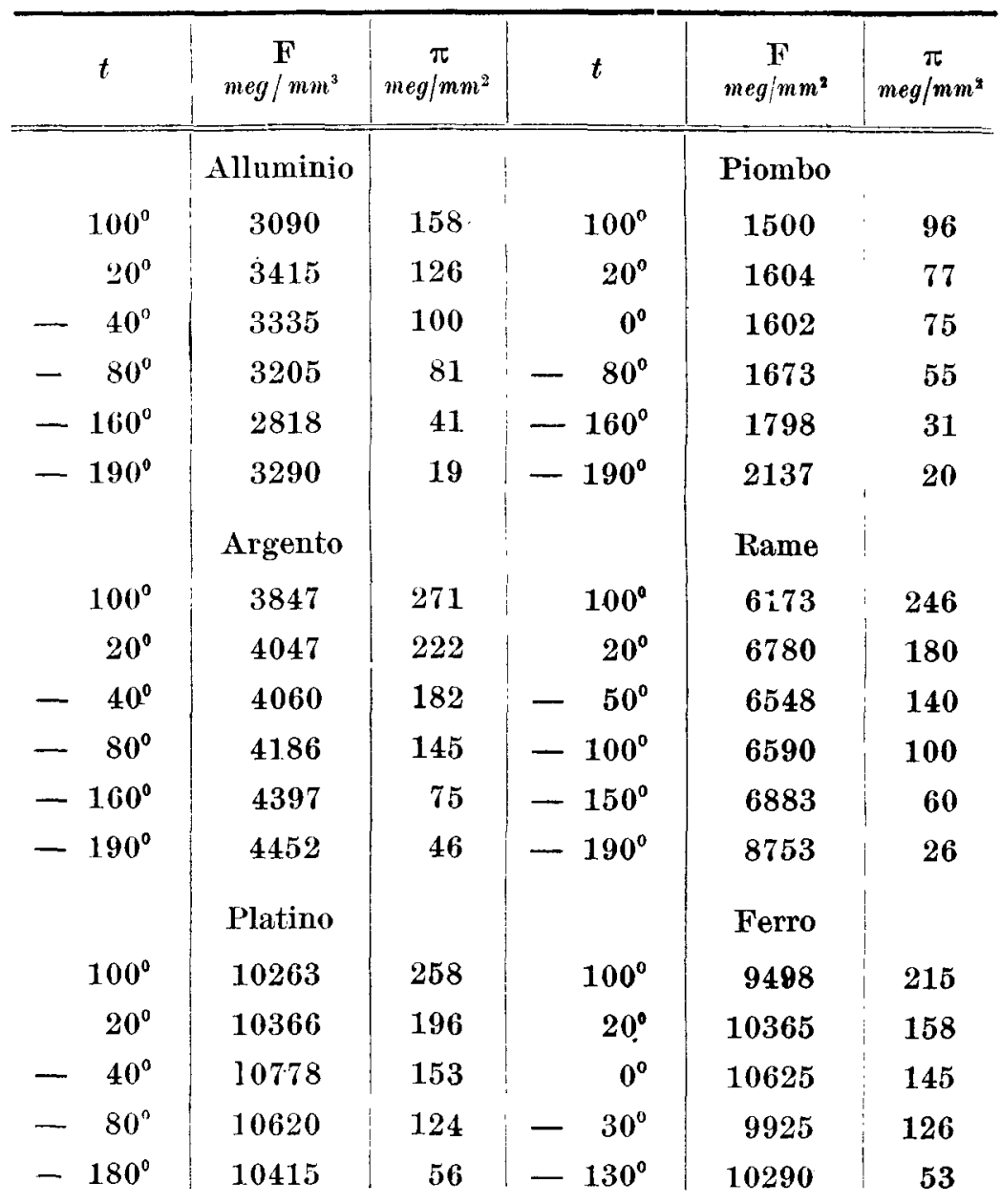


Come si vede dai valori di $\mathbf{F}$, l'andamento della loro variazione con la temperatura, se è lo stesso per tutti i metalli indicati per le temperature superiori all'ordinaria, cioè vanno diminuendo col crescere di essa, diversamente si comportano per temperature inferiori all'ordinaria.

Come risultato generale noi abbiamo che la forza molecolare di coerenza può diminuire tanto con un aumento che con una diminuzione di temperatura, e queste variazioni seguendo quelle del rapporto $\frac{c}{\gamma}$ ci portano a concludere che la variazione di $\mathrm{F}$ non deve dipendere da variazioni di distanza fra le molecole dei corpi dovute a variazioni di temperatura, poichè $\gamma$ cresce continuamente al crescere di questa. Mentre noi abbiamo dalle formole del calore specifico di Einstein e di Nernst e Lindemann che esso è funzione di $\frac{\beta \nu}{T}$ e cioè del rapporto fra la frequenza del moto vibratorio molecolare dei solidi e la temperatura assoluta.

Potrebbero quindi le variazioni della frequenza, ossia dellampiezza delle vibrazioni, spiegare le variazioni corrispondenti della forza molecolare di coerenza.

Quanto al valore della pressione interna esso diminuisce continuamente col diminuire della temperatura e dei valori di $\gamma$ e di $\mu$; andamento, cui ho già sopra accennato, e che conferma il significato dato a tale forza.

$L$ 'indicato comportamento dei valori di $F$ al variare della temperatura trova riscontro in quello analogo dei diversi moduli di elasticità. Difatti per le temperature superiori alla ordinaria anche questi diminuiscono col crescere della temperatura, come hanno dimostrato le misure di Wertheim, Pisati, Benton, Kupfer, Winkelmann. Benton '), applicando le formole della termodinamica alla deformazione per trazione, accompagnata da riscaldamento, giunse all'espressione seguente per la

1) Phys. Rew., 1902. 
variazione del modulo $E$ al variare della temperatura:

$$
\frac{\partial \mathrm{E}}{(\partial \mathrm{T})_{\mathrm{P}}}=-\mathrm{E}^{*}\left(\frac{\partial a}{\partial \mathrm{P}}\right)_{\mathrm{T}}
$$

in cui $a$ è il coefficiente di dilatazione termica lineare e $\mathbf{P}$ la tensione a cui è sottoposto il filo metallico. Detta espressione, quantunque algebricamente rappresenti il senso della variazione di $\mathrm{E}$, non è però verificata dai risultati sperimentali.

La (13), risultando che $\mathrm{F}$, come pure $\sigma$, diminuiscono col crescere della temperatura sopra la ordinaria, porta a concludere che $\mu$ deve crescere colla temperatura, ciò che è dimostrato dalle misure di Gruineisen.

Anche per le temperature più basse dell'ordinaria si riscontrano analogie fra il comportamento dei valori di F, e quelle dei diversi moduli di elasticità. Già Wertheim aveva osservato che i metalli portati a basse temperature $\left(-15^{\circ}\right)$ presentano un effetto residuo, simile a quello dovuto alla ricottura, quando essi vengono riscaldati. Ora questo effetto consisterebbe in una diminuzione del valore di $\mathrm{E}$; che corrisponde ad una diminuzione di $\mathbf{F}$.

L'osservazione fatta dal Benton per il modulo di Young del rame, che, mentre secondo le misure di Wertheim diminuisce del $33 \%$ passando da $15^{\circ}$ a $200^{\circ}$, secondo quelle del Benton diminuisce solo del $18 \%$ passando da $-186^{\circ}$ a $20^{\circ}$, trova appunto la sua spiegazione nelle variazioni del valore di F a temperature sotto la ordinaria, poichè si avrebbe un minimo verso - $50^{\circ}$. Cosi si spiegano pure altre osservazioni del Benton riguardo al modùlo di scorrimento del rame, al modulo di rigidità del rame e dell'acciaio, assimilando il comportamento di questo a basse temperature a quello del ferro. Anzi questo servirebbe a corroborare la spiegazione data dal Benton del fatto che, mentre con un rapido abbassamento di temperatura si ricuoce il rame e si tempra l'acciaio, il modulo di rigidita di questo cresce meno rapidamente di quello del rame andando a basse tem- 
perature. Benton per spiegare questo fatto ricorre ai risultati delle misure di Gray, secondo le quali il detto modulo dell'acciaio invece di aumentare, diminuisce leggermente con la tempra, e che quindi anche l'acciaio non è temprato, ma ricotto, la un subitaneo raffreddamente al disotto della temperatura ordinaria. Quindi egli annuncia un annealing effect dell'aria liquida. Questo si spiega molto bene mediante l'andamento dei valori della forza intermolecolare di coerenza a basse temperature.

Durezza. - Mi resta ancora a fare un cenno della durezza, come proprietà dei solidi, che deve stare in stretta relazione con la forza di coerenza. Si sa che per la durezza non si possono avere valori assoluti e nemmeno che si possano confrontare a rigore fra di loro. Tuttavia si possono riunire fra loro gli elementi in gruppi, i cui costituenti presentino numeri di durezza della scala di Rydberg poco differenti, di cui si calcola un valore medio e paragonare questi valori medii coi corrispondenti medii della forza di coerenza, come nello specchio seguente :

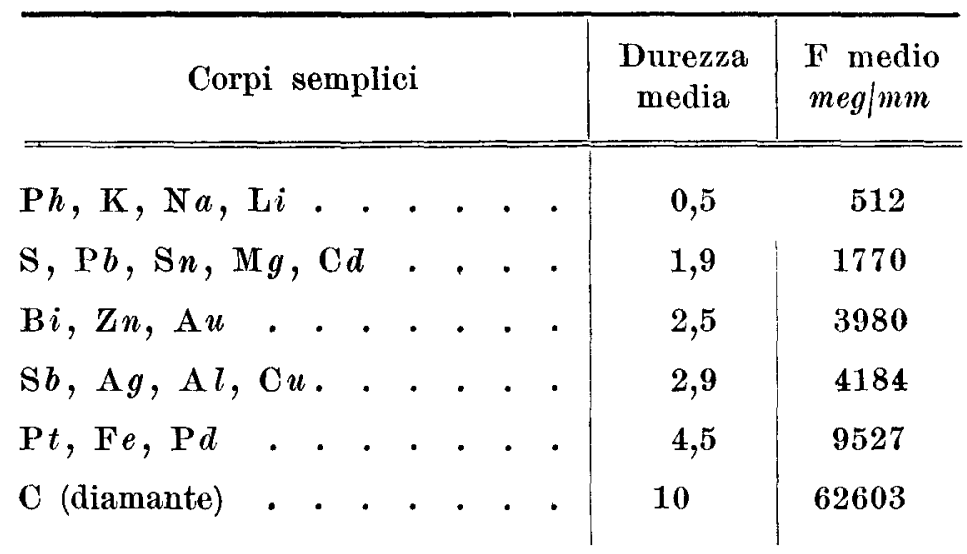

Meno esplicita risulta la relazione fra $\mathrm{i}$ valori di $\mathrm{F}$ ed $\mathrm{i}$ risultati ottenuti per la durezza col metodo della biglia di acciaio temprato di Kohn e Brinell, ma sull'attendibilità dı esso esistono ancora delle forti divergenze di opinioni, il che 
viene spiegato dalle conclusioni, a cui giunge H. Lorenz nella sua teoria della durezza ").

\section{Valori di $f$.}

it la forza molecolare, che entra come fattore del lavoro interno nel cambiamento di stato di aggregazione, nel passaggio di fase, nella fusione dei solidi. Essa può essere calcolata o con la formola (1) o con la (8).

Per pochi corpi abbiamo i valori di $\frac{d p}{d t}$ determinati direttamente. Essi sono dovuti per $\mathrm{Cd}, \mathrm{Hg}, \mathrm{S} n, \mathbf{P} b, \mathrm{~B} i$, a T. Johnson e L. H. Adams, per il fosforo a Lussana, per lo zolfo a Tammann. Nella tabella seguente sono indicate le temperature assolute di fusione, i valori di $\frac{d t}{d p}$, quelli di $f$ espressi in megadine per $\mathrm{cm}^{2}$; nell' ultima colonna sono per confronto indicati i corrispondenti, calcolati con la (8), che verranno in seguito riportati con gli altri. I valori indicati con asterisco sono quelli dovuti a Tammann.

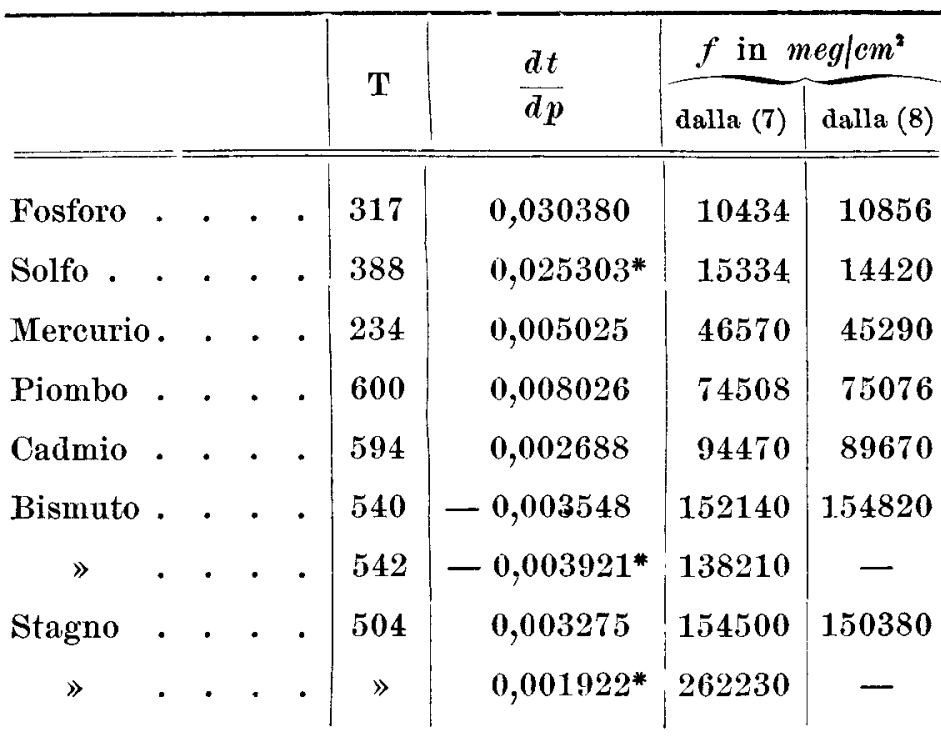

1) Loe. eit., pag. 636 . 
Come si vede dalle ultime due colonne i due valori di $f$ presentano fra loro una buona concordanza. Solo quelli per $\mathrm{B} i$ e $\mathbf{S} n$, ottenuti coi risultati di Tammann, si presentano del tutto discordanti.

Vengo ora ai valori di $f$ calcolati colla (8). I valori della temperatura di fusione $\mathrm{T}$ e del calore di vaporizzazione $r$ sono gli stessi da me adottati nello studio sull'entropia nei corpi semplici (3), compreso quello di $r$ del ferro ivi calcolato. Cosi pure parte dei valori della variazione di volume per grammo nella fusione furono dedotti dai valori di $\Delta v$ ivi adottati. Altri invece sono dovuti al Toepler e sono indicati con asterisco; quello del tallio a Omodei, e quello del ferro a Wrighton o Roberts. I corpi semplici sono distinti in metalli e non metalli e si seguono secondo l'ordine crescente dei valori di $f$. Si assume $p=\mathrm{I}$. 
SULLE FORZE MOLECÒLARI INTERNE DEI CORPI ECG. 125

\begin{tabular}{|c|c|c|c|c|c|}
\hline ME'TALL & & $\mathbf{T}$ & $\begin{array}{c}r \\
c a l \mid g\end{array}$ & $\begin{array}{c}\Delta v \\
\mathrm{~cm}^{3} / g\end{array}$ & $\begin{array}{c}f \\
m e g / \mathrm{cm}^{2}\end{array}$ \\
\hline Rubidio. & - & 311,5 & 6,1 & $0,0140^{*}$ & 18252 \\
\hline Potassio & . & 335 & 13,6 & 0,0286 & 19908 \\
\hline Sodio . & . & 371 & 17,75 & 0,0213 & 24906 \\
\hline Mercurio & . & 234 & 2,8 & 0,0026 & 45290 \\
\hline Litio. & . & 453 & 32,8 & 0,0262 & 52428 \\
\hline Piombo. & . & 597,5 & 5,5 & 0,0031 & 75076 \\
\hline Tallio & - & 563 & $\tau, 2$ & 0,00376 & 80215 \\
\hline Cadmio . & . & 594 & 13,7 & 0,0060 & 95620 \\
\hline Zinco . & - & 688 & 28,1 & $0,0105^{*}$ & 112110 \\
\hline Stagno . & - & 503 & 14,0 & $0,0039^{*}$ & 150380 \\
\hline Alluminio & . & 898 & 100,0 & 0,0205 & 204600 \\
\hline Ferro . & . & 1805 & 57,8 & $-0,0085$ & -285510 \\
\hline NON META & 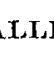 & & & & \\
\hline Fosforo & . & 317 & 5,0 & 0,0193 & 10856 \\
\hline Iodio & . & 386 & 11,6 & $0,0434^{*}$ & 11196 \\
\hline Bromo . & . & 266 & 16,2 & $0,0511^{*}$ & 13264 \\
\hline Solfo. . & . & 390 & 9,9 & $0,0287^{*}$ & 14420 \\
\hline Tellurio. & . & 728 & 19,0 & $0,0123^{*}$ & 64707 \\
\hline Antimonio & & 905 & 40,2 & $0,0022^{*}$ & 76546 \\
\hline Bismuto & . & 540 & 12,6 & $-0,0034$ & -154820 \\
\hline
\end{tabular}

Se si confrontono i valori di $f$, dati dalla precedente tabella con quelli di $\mathrm{F}$, si vede che i primi crescono nello stesso ordine di grandezza che i secondi, il che dimostra esservi 
analogia sulla natura di quelle due forze. Partendo dalla (2) e dalla (8) si deduce che il rapporto fra $\mathrm{F}$ e $f$ dipende principalmente dal rapporto fra il calore specifico a volume costante ed il calore di vaporizzazione interno, equivalenti ambedue a variazioni della energia interna del corpo, rispettivamente nulla trasformazione isometrica di stato fisico e nel cambiamento isotermico di fase. Sul valore di $F$ devo però influire la natura chimica delle molecole, ed anche su quello di $f$.

ì da notare che il valore di $f$ per ciascun corpo è molto minore di quello di $F$, e che per il ferro ed il bismuto, avendosi una diminuzione di volume nella fusione, corrispondono valori negativi di $f$. Ora siccome questa grandezza deve presentare delle variazioni analoghe alla $F$ col variare della temperatura, così può darsi che alla temperatura di fusione per il bismuto ed il ferro abbia raggiunto valori negativi. Difatti se per i tre metalli $\mathrm{Pb}, \mathrm{A} l, \mathrm{~F} e$, mediante un'estrapolazione, alquanto arbitraria, dei valori di $\mathbf{F}$ a temperatura superiori a quella di $100^{\circ}$, cerchiamo di calcolare $F$ alle rispettive temperature di fusione, troviamo ancora valori positivi per $\mathrm{Pb}$ e $\mathrm{Al}$, ma negativo per il ferro.

Inoltre la $f$ essendo espressa da $\mathrm{T} \frac{d p}{d t}-p$, se ne deduce che col crescere della temperatura deve diminuire $-\frac{d p}{d t}$, e cioè orescere la variazione della temperatura di fusione per aumento di pressione.

\section{Conclusioni.}

Da quanto sopra esposto si può arrivare alle seguenti conclusioni.

Anzitutto possiamo considerare e calcolare il valore di tre specie di forze molecolari interne nei solidi:

l'una, che chiamiamo forza intermolecolare di coerenza, che entra come fattore nei lavori interni in cui si può trasformare la energia termica, oppure l'energia meccanica, senza variazione di volume del corpo; 
la seconda, di natura analoga alla pressione esterna, e che chiamiamo pressione interna, che entra come fattore nei lavori interni, in cui si impiega energia termica con variazione di volume del corpo;

la terza, che entra come fattore nei lavori interni, compiuti nei cambiamenti di fase, nella fusione di un solido, e che si potrebbe chiamare forza intermolecolare di cambiamento di fase. Sembra che questa terza forza, che agisce alla temperatura di fusione, alla temperatura cioè, alla quale, secondo Lindemann, le ampiezze delle oscillazioni atomiche intorno alla loro posizione di equilibrio diventano dello stesso ordine di grandezza della distanza media fra gli atomi, sia della stessa natura della forza $\mathrm{F}$, che agisce con valori particolari alle diverse temperature.

La grandezza della prima forza presenta delle relazioni interessanti coi valori di talune grandezze considerate nello studio e nelle applicazioni delle proprietà elastiche dei solidi. Le più semplici di queste relazioni di $F$ coi moduli di elasticità di trazione $\mathrm{E}$, di rigidità $n$, di scorrimento $\mathrm{N}$ sareb). bero le seguenti : $\mathrm{E}=1,70 \mathrm{~F}$ od approssimativamente

$$
\mathbf{E}=\frac{5}{3} \mathrm{~F} ; \quad n=\frac{\mathrm{F}}{\pi} \quad \mathrm{N}=\frac{2}{\pi} \mathrm{F} .
$$

Il relativamente grande rapporto fra $\mathrm{E}$ ed $\mathrm{F}$ dipende da che il carico, rappresentato da $\mathrm{E}$, e che dovrebbe raddoppiare la lunghezza iniziale del solido, non è che un carico ideale, poichè nella sua applicazione, molto prima che sia raggiunto quell'effetto, si oltrepassa non solo il limite di elasticità, ma anche il carico di rottura o tenacità. Riterrei quindi più conforme ad esattezza introdurre nelle formole della tecnica dell'elasticità la grandezza $\mathrm{F}$, che è una grandezza reale, che si può calcolare alle diverse temperature, mediante grandezze fisiche esattamente determinate, invece di $\mathrm{E}$, che è una grandezza puramente ideale. Facendo questa sostituzione si arriva alle seguenti espressioni approssimate: 
Modulo di compressione uniforme o di elasticità cubica

$$
K=\frac{5 \underline{F}}{9(1-2 \sigma)} \text {. }
$$

Modulo di scorrimento

$$
\mathrm{N}=\frac{5 \mathrm{~F}}{6(1+\sigma)}
$$

Modulo di compressione secondo lo spessore

$$
\mathrm{K}^{2}=\frac{5(1-\sigma) \mathrm{F}}{3(1+\sigma)(1-2 \sigma)}
$$

Uoefficiente oi allungamento per trazione

$$
\alpha=\frac{3}{5 \mathrm{~F}}
$$

Ooefficiente di Lamè

$$
\lambda=\frac{5 \sigma \mathrm{F}}{3(1+\sigma)} \frac{}{(1-2 \sigma)} .
$$

Coefficiente di contrazione trasversale

$$
\beta=\frac{3 \sigma}{5 \mathrm{~F}}
$$

Coefficiente di compressibilità cubica

$$
\mu=\frac{9(1-2 \sigma)}{5 \mathrm{~F}}
$$

Se si adotta per il coefficiente di Poisson il valore $\sigma=\frac{1}{3}$ che risulta come medio dai dati sperimentali, e che abbiamo veduto potersi considerare come costante caratteristica per i solidi isotropi, tutte quelle espressioni si trasformano nelle seguenti :

$$
\begin{aligned}
\mathrm{E}=\mathrm{K}=\frac{5 \mathrm{~F}}{3} & \mathrm{~N}=\frac{5 \mathrm{~F}}{8} & n=\frac{5 \mathrm{~F}}{16} \\
\mathrm{~K}^{\prime}=\frac{5 \mathrm{~F}}{2} & \lambda=\frac{5 \mathrm{~F}}{4} & \alpha=\mu=\frac{3}{5 \mathrm{~F}} \quad \beta=\frac{1}{5 \mathrm{~F}} .
\end{aligned}
$$


SULLE FORZE MOLECOLARI INTERNE DEI CORPI ECC. 129

Quindi possiamo arrivare alle seguenti conclusioni generali: I moduli di elasticità per qualunque specie di deformazione. sono direttamente proporzionali alla forza molecolare di coerenza.

I coefficienti caratteristici di qualunque specie di deformazione elastica sono inversamente proporzionali alla forza mo. lecolare di ooerenza.

Palermo, ottobre 1917. 